\title{
The Pursuit of Engineering the Ideal Heart Valve Replacement or Repair: A Special Issue of the Annals of Biomedical Engineering
}

Heart valve disease is a major component of heart disease and the significance of this disease is rising primarily due to increasing life expectancy as well as new percutaneous treatments becoming available to older patient populations who would otherwise only receive medical management. Treatments for severe heart valve disease vary from replacement with prosthetic heart valves or performing repair or implantation of palliative devices to improve valvular function. Unfortunately, current and past replacement and repair devices have not "solved" the problem due to continuing issues related to occurrence of complications (e.g. thromboembolism, need for pacemaker etc.) or compromise of valve function (e.g. structural deterioration of prosthetic leaflets, recurrence of mitral regurgitation after repair, valve leakage etc.) or the unintended introduction of a new problem (e.g. new issues resulting from the need for anticoagulation therapy). This explains the strong need and the current pursuit of engineering the ideal heart valve replacement or repair, which is the focus of this special edition. Two review articles are presented: (a) review by Dasi et al. on the mechanics of transcatheter aortic valve replacement (TAVR) which focuses on the current issues related to $\mathrm{TAVR}^{4}$; and (b) the review by Espiritu et al. which focuses on trans-catheter mitral valve repair therapies. ${ }^{5}$ We have limited the reviews to transcatheter approaches alone for the aortic and mitral technologies given that numerous other review articles exist on the more traditional heart valve replacement and repair technologies.

So what would be the ideal heart valve replacement or repair solution? While the best solution is prevention of valve disease, we will not be discussing preventative approaches in this edition because that would entail engineering in a different sense - understanding the pathophysiology of valve disease and engineering an intervention aimed to arrest or reverse disease progression. Here we focus on engineering new devices that will overcome the limitations of current replacement or repair devices. Two broad strategies emerge: (A) engineer a living tissue engineered heart valve that can replace the diseased heart valve and be immune to deterioration or disease processes or (B) engineer replacement or repair devices that are not biological in nature to overcome the limitations of current devices.

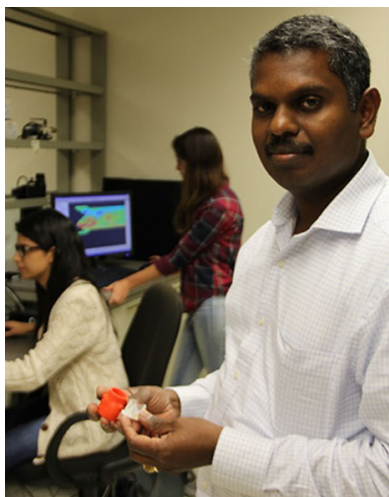

Lakshmi Prasad Dasi

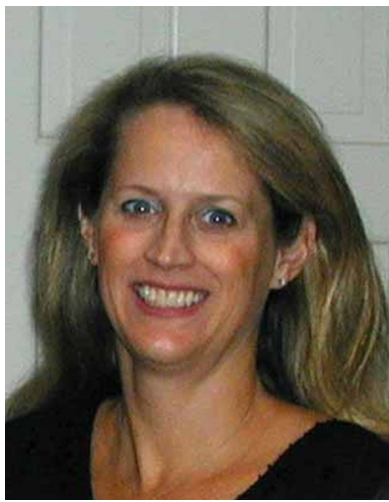

Karyn Kunzelman

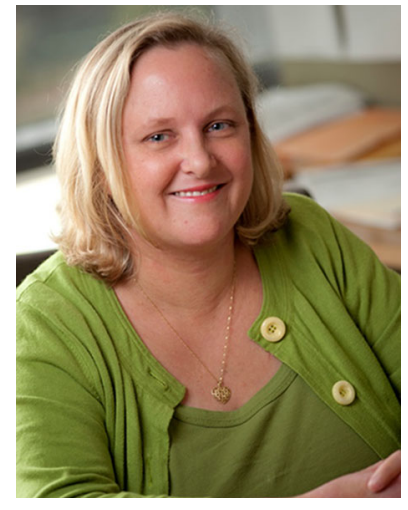

Jane Grande-Allen

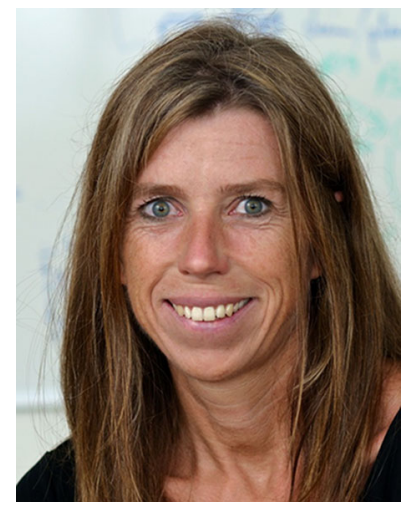

Ellen Kuhl
Both strategies continue to be challenging because the mechanics of heart valves is inherently complex and unfortunately the heart valve engineering community does not yet fully understand the principles dictating how living tissue responds to mechanics or how a given valve design with novel synthetic materials would perform in vivo. Basic studies addressing this knowledge gap is progressing and a few articles are noteworthy along these efforts. The work presented by Kang et al. contributes to the body of knowledge that aims to $3 \mathrm{D}$ print cardiovascular soft tissues such as heart valves. ${ }^{7}$ Here they have elucidated the importance of stress mechanisms in the context of optimizing photo-encapsulation viability of heart valve cells for 3D printing applications. The article by Khalighi et al. aims to take advantage of regularities in the mitral valve structure to improve current mitral valve repair 
strategies. ${ }^{8}$ New basic understanding of the limitation in durability as well as poor aortic sinus flow patterns among current TAVR devices is the focus of the articles by Martin and Sun and Midha et al. respectively. ${ }^{9,10}$

A variety of innovative tissue engineering strategies are being employed for the development of living heart valve replacements. A primary concern at the present is how to translate these new strategies successfully to the clinic. Four different approaches are described in this special issue, each addressing a unique hurdle that must be overcome in order to advance the field of heart valve tissue engineering and make these technologies realistic options for patients. Although the work of Kang et al. employs a more basic science framework to study cell viability, their optimization of crosslinking conditions during the encapsulation of cells is a necessary step in the assessment of hydrogels as a viable scaffold material for tissue engineered heart valves (TEHVs). Alavi et al. have taken a different approach in their efforts to improve the durability of tissue engineered valves through the incorporation of a superelastic nitinol mesh into a hybrid living valve. ${ }^{1}$ The articles by Picard-Deland et al. and Reimer et al. both focus on tubular heart valves. ${ }^{11,12}$ Tissue engineered tubular heart valves are emerging as an attractive approach with potential for growth-a characteristic critical for pediatric applications. These two papers offer contrasting approaches to combat the major hurdle of post-implantation activation of valve cells within the TEHV and subsequent contraction of the engineered tissue. Picard-Deland et al. allows a precontraction phase during the in vitro culture of novel tubular valves derived from self-assembled cell/matrix layers, whereas Reimer et al. decellularize the tubular scaffold prior to implantation within a preclinical lamb model.

With regards to the more traditional approaches of developing non biological devices that promise to overcome the limitations of current replacement and repair devices, we note four articles. While the article by Bark Jr. et al. focuses on overcoming the limitations of mechanical heart valves through the incorporation of superhydrophobic materials in order to eliminate the need for anticoagulation therapy, ${ }^{2}$ the articles by Yousefi et al. focus on novel tri-leaflet synthetic heart valves made from polymeric or textile (fabric) heart valves. ${ }^{14,15}$ Both approaches tackle the issue of developing a non-biological alternative valve which would be both durable and free from anticoagulation therapy. Bark et al.'s study shows that by making the mechanical heart valve's surface superhydrophobic, the physical contact area between blood and the valve's surfaces can be dramatically reduced effectively relaxing the no-slip fluid boundary condition resulting in reduced skin drag and wall shear stresses. On the other hand, Yousefi et al.'s articles aim to overcome the limited durability of bioprosthetic tissue by constructing tri-leaflet heart valve prosthesis using synthetic materials such as biomolecule enhanced polymers or fabric. These studies illustrate that the design of the valve is not independent of the leaflet material and instead highlight the need to further understand the principles dictating valve design for a given material. The article by $\mathrm{He}$ et al. focuses on a novel approach to treat mitral regurgitation with the introduction of a trans-apical coaptation plate, offering a potentially simple procedure compared to current mitral valve repair options. ${ }^{6}$

Finally, we highlight the need for new engineering modeling approaches that will help accelerate the pursuit of the ideal heart valve replacement or repair. Throughout the past decade, mathematical modeling and computational simulation have advanced as essential technologies to quantify the state of valve disease, identify the optimal treatment strategy, and predict the outcome of a specific valve repair and replacement technique. Advancements in medical imaging have brought us closer than ever to characterize the biomechanics of heart valves in their in vivo state and precisely quantify deformation, strain, stress, pressure, and flow conditions in the living heart. This special issue highlights the current trend to integrate all this information-fluid flow and structural deformation-into a single fluid-structure-interaction model. The moving boundaries of the heart valve leaflets under complex flow conditions make this an exceptionally difficult problem and a number of new strategies are currently emerging to address these challenges. This special issue summarizes state-of-the-art technologies in modeling and simulation and illustrates that these techniques - previously just considered for merely scientific purposes - are now increasingly integrated in clinical decision making. Special emphasis is placed on the need for strong experimental validation for the ever increasingly complex heart valve simulations, which in itself can be challenging. The article by Bloodworth IV et al. illustrates this paradigm with the development of ex vivo methods for not only evaluating the sensitivity of computational methods to boundary conditions but also to serve the purpose of developing validation datasets for future computational models. ${ }^{3}$ Naturally, an accurate geometric representation of the valve forms the basis for a successful simulation. Like in almost any other medical field, we are currently witnessing an unstoppable trend towards personalized modeling, based on individual geometric models from a patients' own heart. Not only basic scientists, but also clinical researchers and medical practitioners are recognizing the potential of person- 
alized simulation to identify a specific device or a particular surgical procedure with the best possible outcome for each individual patient. Recent trends suggest to not only utilize these geometric models for biomechanical analyses, but also to use them directly to create three-dimensional, anatomically exact, personalized models via three-dimensional printing. This is illustrated well in the article by Vukicevic et al. where patient specific geometric representations is shown to be critically important using 3D printing technologies when assessing the efficacies of structural interventions. $^{13}$ Three-dimensional printing is a powerful technology that is increasingly recognized for patient illustration, surgical training, and treatment planning. It already allows us to create personalized solutions for heart valve repair, and has the potential to enable personalized tissue engineered solutions for heart valve replacement.

\section{REFERENCES}

${ }^{1}$ Alavi, S. H., M. S. Baliarda, N. Bonessio, L. Valdevit, and A. Kheradvar. A tri-leaflet nitinol mesh scaffold for engineering heart valves. Ann. Biomed. Eng. 2016. doi: 10.1007/s10439-016-1778-0.

${ }^{2}$ Bark D. L., H. Vahabi, H. Bui, S. Movafaghi, B. Moore, A. K. Kota, K. Popat, and L. P. Dasi. Hemodynamic performance and thrombogenic properties of a superhydrophobic Bileaflet mechanical heart valve. Ann. Biomed. Eng. 2016. doi:10.1007/s10439-016-1618-2.

${ }^{3}$ Bloodworth, C. H., E. L. Pierce, T. F. Easley, A. Drach, A. H. Khalighi, M. Toma, M. O. Jensen, M. S. Sacks, and A. P. Yoganathan. Ex vivo methods for informing computational models of the mitral valve. Ann. Biomed. Eng. 2016. doi:10.1007/s10439-016-1734-z.

${ }^{4}$ Dasi, L. P., H. Hatoum, A. Kheradvar, R. Zareian, S. H. Alavi, W. Sun, C. Martin, T. Pham, Q. Wang, P. A. Midha, V. Raghav and A. P. Yoganathan. On the mechanics of transcatheter aortic valve replacement. Ann. Biomed. Eng. 2016. doi:10.1007/s10439-016-1759-3.

${ }^{5}$ Espiritu, D., D. Onohara, K. Kalra, E. L. Sarin, and M. Padala. Transcatheter mitral valve repair therapies: evolution, status and challenges. Ann. Biomed. Eng. 2016. doi: 10.1007/s10439-016-1655-x.

${ }^{6} \mathrm{He}, \mathrm{Z}$., K. Zhang, T. Jing, and Y. Wang. Transapical coaptation plate for functional mitral regurgitation: an in vitro study. Ann. Biomed. Eng. 2016. doi: 10.1007/s10439-016-1726-z.

${ }^{7}$ Kang, L. H., P. A. Armstrong, L. J. Lee, B. Duan, K. H. Kang, and J. T. Butcher. Optimizing photo-encapsulation viability of heart valve cell types in $3 \mathrm{~d}$ printable composite hydrogels. Ann. Biomed. Eng. 2016. doi:10.1007/ s10439-016-1619-1.

${ }^{8}$ Khalighi, A. H., A. Drach, C. H. Bloodworth IV, E. L. Pierce, A. P. Yoganathan, R. C. Gorman, J. H. Gorman III, and M. S. Sacks. Mitral valve chordae tendineae: topological and geometrical characterization. Ann. Biomed. Eng. 2016. doi:10.1007/s10439-016-1775-3.

${ }^{9}$ Martin, C., and W. Sun. Transcatheter valve underexpansion limits leaflet durability: implications for valvein-valve procedures. Ann. Biomed. Eng. 2016. doi: 10.1007/s10439-016-1738-8.

${ }^{10}$ Midha, P. A., V. Raghav, I. Okafor, and A. P. Yoganathan. The effect of valve-in-valve implantation height on sinus flow. Ann. Biomed. Eng. 2016. doi:10.1007/ s10439-016-1642-2.

${ }^{11}$ Picard-Deland, M., J. Ruel, T. Galbraith, C. Tremblay, F. Kawecki, L. Germain, and F. A. Auger. Tissue-engineered tubular heart valves combining a novel precontraction phase with the self-assembly method. Ann. Biomed. Eng. 2016. doi:10.1007/s10439-016-1708-1.

${ }^{12}$ Reimer, J., Z. Syedain, B. Haynie, M. Lahti, J. Berry, and R. Tranquillo. Implantation of a tissue-engineered tubular heart valve in growing lambs. Ann. Biomed. Eng. 2016. doi: 10.1007/s10439-016-1605-7.

${ }^{13}$ Vukicevic, M., D. S. Puperi, K. J. Grande-Allen, and S. H. Little. 3d printed modeling of the mitral valve for catheterbased structural interventions. Ann. Biomed. Eng. 2016. doi:10.1007/s10439-016-1676-5.

${ }^{14}$ Yousefi, A., A. Vaesken, A. Amri, L. P. Dasi, and F. Heim. Heart valves from polyester fibers vs. biological tissue: comparative study in vitro. Ann. Biomed. Eng. 2016. doi: 10.1007/s10439-016-1632-4.

${ }^{15}$ Yousefi, A., D. L. Bark, and L. P. Dasi. Effect of arched leaflets and stent profile on the hemodynamics of tri-leaflet flexible polymeric heart valves. Ann. Biomed. Eng. 2016. doi:10.1007/s10439-016-1674-7.

LaKshmi Prasad Dasi

The Ohio State University

Columbus, OH, USA

Electronic mail: dasi.1@osu.edu

JANe GRANDe-Allen

Rice University, Houston, TX, USA

KARYN KUnZelman

University of Maine, Orono, ME, USA

ELLEN KUHL

Stanford University, Stanford, CA, USA 